\title{
Examination of the Usefulness of the Obstacle-Single Leg Forward Step (OSFS) Test for Evaluating Fall Risk
}

\author{
Sohee Shin ${ }^{1}$, Shinichi Demura ${ }^{2}$, Toshiro Sato ${ }^{3}$ \\ ${ }^{1}$ Center for Innovation Venture Business Laboratory, Kanazawa University, Kanazawa, Japan; \\ ${ }^{2}$ Graduate School of Natural Science and Technology, Kanazawa University, Kanazawa, Japan; \\ ${ }^{3}$ Department of Health and Sports, Health and Welfare, Niigata University, Niigata, Japan. \\ Email: sohee@ed.kanazawa-u.ac.jp \\ Received October 12 $2^{\text {th }}, 2011$; revised November 15 $5^{\text {th }}, 2011$; accepted November $24^{\text {th }}, 2011$.
}

\begin{abstract}
This study aimed to examine the usefulness of the Obstacle-Single leg forward step (OSFS) test for evaluating fall risk and fall-related physical fitness. One hundred and eighty four older women were divided into fallers (n $=47$ ) who had a fall experience within the last year and non-fallers who had not $(\mathrm{n}=137)$. In the OSFS test, they stepped forward over an obstacle and returned to their original position five times as quickly as possible. The OSFS test was also examined in the following divided phases: the OSFS-F phase in which participants stepped out and the OSFS-R phase in which they returned to the stepped leg. A mean time of five steps was used for analysis. In addition, the 10-meter walk and one leg standing with open eyes tests were conducted and the better times were used for analysis. The times of the non-fallers were shorter in the OSFS test, 10-meter walk time, and were longer in the one-leg standing time than those of the fallers (p-value: OSFS 0.025, 10-meter 0.046 , One-leg standing time 0.028). A significant difference was found only in the OSFS-R, the non-fallers showed shorter time than the fallers (p-value: OSFS-F 0.354, OSFS-R 0.010). The OSFS test can discriminate the difference between fallers and non-fallers with the same accuracy as the 10-meter walk time and one-leg standing time tests.
\end{abstract}

Keywords: The Elderly, Faller/Non-Faller, Fall

\section{Introduction}

Falls in the elderly generally result in more serious injury. Some studies reported that falls frequently occur when turning around during walking, when initiating walking (Mano, 2008), or when tripping or stumbling over something. When people feel that they are falling, they generally try to regain their balance with a step reset (taking a step) or a jump reset (Hosoda, 2001). However, the elderly, as opposed to the young, often fall because of delayed response time (Tanaka et al., 2001).

A decline in physical fitness with age is a limiting factor in successful completion of activities of daily living, and it also enhances the possibility of falls among elderly people. In order to adequately evaluate the physical fitness level of the elderly, it will be important that they complete the requirements of daily life independently without a fall. Suzuki et al. evaluated the ADL ability and physical functions of community-dwelling elderly using the Tokyo Metropolitan Institute of Gerontology Index, and they reported that the fallers' score was higher than that of the non-fallers (Suzuki, 2003; Suzuki, 2000). Tinetti (1986) also reported that elderly people with a high activity level have a lower incidence of falls. Hence, a decline in the ability to perform activities of daily living may closely correspond to the fall risk (Tinetti, 1986).

Until now, some studies on fall prediction have been performed using responses to stepping out against various disturbance condtions (Rogers et al., 2001; Hsiao-Wecksler, 2007; Whitney et al., 2007). Because these studies impose disturbance stimulations directly on the bodies of the elderly, they may indeed indicate a fall risk.

Humans avoid a fall and return to a stable base of support (BOS) by stepping forward quickly when sensing an impending fall. Shin and Demura (2007, 2009a, 2009b, 2010) focused on this quick forward step by developing the Single leg forward step test (SFS test) which evaluates the usefulness of the elderly's fall-related physical fitness. Because, in the single-leg forward step test, participants must step forward and then return to their original position quickly, considerable strength and balance are both required in the supporting and stepping legs. It was reported that the test using this movement mirrors the fall risk score (Demura et al., 2010) and is useful for evaluating the elderly's fall-related physical fitness (Shin \& Demura, 2010).

Factors associated with falls are divided into two categories the inner factor caused by the intrinsic body and the extrinsic factor caused by a person's environment. These factors relate singly or multiply to falls. Although incidents of falls included "Slipping", "Reel", "Missing a step", "Trip over", etc., the main cause of falls among the elderly was tripping over an obstacle or a step (Saito \& Muraki, 2010).

Vandervoot et al. (1989) reported that the elderly experience falls more often because a sensory and motor function related to postural and balancing maintenance during walking decreases with age. Mano (2008) reported that the elderly are inferior in standing and walking abilities to young people and the decline in these abilities relates closely to the fall risk. A decline in physical functions and walking ability with age has been considered to be the primary cause (Aoyagi, 2001). As suggested by the above findings, it may be effective to add an obstacle to the traditional Single leg Forward step test to evaluate the elderly's fall risk when there is the possibility of a trip.

Meanwhile, the balance and walking abilities of the elderly have been evaluated by respective one-leg standing time with open eyes and walking velocity tests (Mano, 2008). A decline in both abilities corresponded closely with fall risk and is related to a decline in sensory and motor functions as well as a change in sensorymotor processing in the central nervous sys- 
tem as people age. We hypothesized that the fallers are inferior to the non-fallers in the Obstacle-Single leg forward step (OSFS) test.

This study aimed to examine the usefulness of the OSFS test to evaluate fall risk and the fall-related physical fitness of the elderly by comparing results on the 10 -meter walk test and one-leg standing with open eyes test.

\section{Methods}

\section{Participants}

The participants were 184 healthy elderly women who can walk independently. Prior to various measurements, the purpose and procedure of this study were explained in detail and informed consent was obtained from all participants. This study was approved by the Kanazawa University Department of Education Ethical Review Board.

Participants were divided into fallers $(n=47)$ with a fall experience over the last year and non-fallers $(n=137)$. Table 1 shows their anthropometric characteristics.

\section{Apparatuses and Methods}

\section{Obstacle-Single Leg Forward Step Test (OSFS Test)}

Participants stood with relaxed arms and barefooted on a step sheet in a quiet room. They were asked to gaze at an obstacle. Before the measurement, participants were evaluated to determine which leg was easiest to stand on and operate by Demura's assessment (Demura et al., 2010). Participants stood with the supporting leg, stepped forward over the obstacle with another leg, and returned to an original position five times as quickly as possible (Figure 1). The step width from the start spot was $25 \mathrm{~cm}$, and the obstacle was set in the center of the distance. The obstacle was 10 centimeters high block. The measurement was conducted in one trial after one practice, and the mean time of five steps was used for statistical analysis. A tester controlled an obstacle lightly so that it did not move from a set place and or fall down even if participants tripped over it. The step test was performed using the step sheet (Takei inc. Japan). This device can measure swing time from one leg rising to landing based on foot pressure information. Parameters were the time of a forwarding phase (OSFS-F: A mean time of stepping forward) and a returning phase (OSFS-R: A mean time of returning to the original place), and a mean time of both phases.

\section{One Leg Standing Time Test}

Participants stood with bare feet and put both hands on their waist. After lifting up one leg according to a signal, a standing time was measured. The test was performed using one trial with each leg. The maximum length of the test was 120 seconds. A longer time of both legs was used for analysis. In addition, the time was measured until one of the following occurred: the lifting foot reached the floor or touched the other leg, the supporting leg's position shifted, or the lumbar hand left the waist.

\section{0-Meter Walk Test}

A 10-meter walk test was conducted on flat ground. A line of $10 \mathrm{~cm}$ width was drawn at the start and finish spots so that participants knew the course of the walk. Participants were instructed to walk as quickly as possible. Using a stopwatch, the tester measured the time between stepping from the starting line $(0 \mathrm{~m})$ to stepping over the goal line $(10 \mathrm{~m})$. A test was conducted twice and the faster time was used as a representative value.

\section{Data Analysis}

Mean differences between the fallers and non-fallers groups
Table 1.

Mean differences of physique characteristics between fallers $(n=137)$ and non-fallers $(n=47)$.

\begin{tabular}{cccccc}
\hline & Group & Mean & SD & t-value & ES \\
\hline Age & Faller & 76.68 & 5.43 & 0.906 & 0.15 \\
(year) & Non-faller & 75.80 & 5.89 & & \\
Height & Faller & 147.55 & 4.86 & 0.448 & 0.07 \\
(cm) & Non-faller & 147.13 & 5.98 & & \\
Weight & Faller & 50.81 & 8.45 & 1.069 & 0.18 \\
$(\mathrm{~kg})$ & Non-faller & 49.37 & 7.81 & & \\
\hline
\end{tabular}

Note: ES: Effect size.

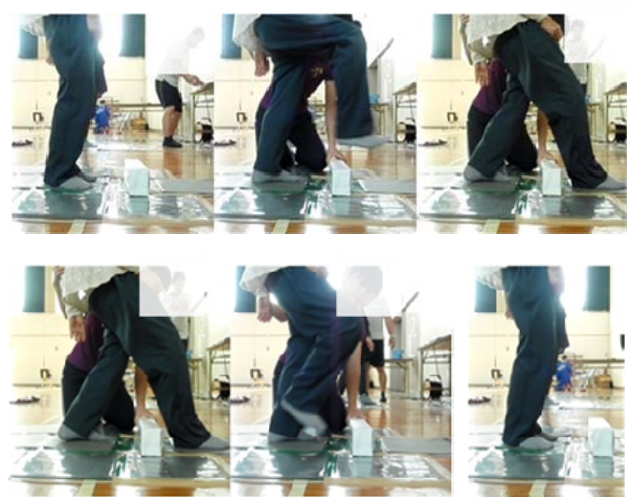

Figure 1.

Obstacle Single leg Forward Step test (OSFS) test. (a) Participants stepped forward while stepping over an obstacle (OSFS-F); $(b)$ and returned to original place while stepping over an obstacle (OSFS-R). Note: Participants repeated the above measurement five times, as fast as possible.

for each selected parameter were tested using an unpaired t-test. To examine the size of mean differences, an effect size (ES) was calculated. The statistical significance level in this study was set at $5 \%$. Statistical program for the calculation was applied IBM SPSS statistics 19.

\section{Results}

Table 1 shows the test results of the mean differences of physique characteristics between fallers and non-fallers. There was no significant difference in age, height and weight between both groups.

Table 2 shows the test results of mean differences between groups (fallers/non-fallers) in each test. The times of the non-fallers were shorter in the OSFS test, 10-meter walk time, and were longer in the one-leg standing time than those of the fallers. A significant difference was found only in the OSFS-R (See 2 and 3 in the method), and the non-fallers had shorter times than did the fallers. In addition, the effect sizes of the OSFS and OSFS-R were more than 0.40 .

\section{Discussion}

To evaluate physical fitness of the elderly, highly safe tests should be selected due to the elderly's inferior physical fitness. In addition, it is desirable that the test content relates closely to their activities of daily living and is available for rehabilitation and functional recovery (Demura et al., 2008). This study examined the usefulness of a new step test which added an Obstacle to the traditional Single leg Forward step test with before and back shift of a body center of gravity to more adequately evaluate the elderly's fall-related physical fitness. This test is practical because it does not require a large place and also su- 
Table 2

Mean differences between Fallers $(n=47)$ and Non-fallers $(n=137)$ in each test.

\begin{tabular}{|c|c|c|c|c|c|}
\hline & Group & Mean & SD & t-value & ES \\
\hline \multirow{2}{*}{ OSFS (sec.) } & Faller & 0.64 & 0.16 & \multirow{2}{*}{2.254} & \multirow{2}{*}{0.40} \\
\hline & Non-faller & 0.59 & 0.13 & & \\
\hline \multirow{2}{*}{ OSFS-F (sec.) } & Faller & 0.60 & 0.16 & \multirow{2}{*}{0.929} & \multirow{2}{*}{0.16} \\
\hline & Non-faller & 0.58 & 0.15 & & \\
\hline \multirow{2}{*}{ OSFS-R (sec.) } & Faller & 0.67 & 0.18 & \multirow{2}{*}{2.596} & \multirow{2}{*}{0.46} \\
\hline & Non-faller & 0.60 & 0.15 & & \\
\hline \multirow{2}{*}{$\begin{array}{l}\text { 10-meter walk } \\
\text { time (sec.) }\end{array}$} & Faller & 6.93 & 1.24 & \multirow{2}{*}{2.013} & \multirow{2}{*}{0.35} \\
\hline & Non-faller & 6.48 & 1.31 & & \\
\hline \multirow{2}{*}{$\begin{array}{l}\text { One leg standing } \\
\text { time with eye open } \\
(\mathrm{sec} .)\end{array}$} & Faller & 20.37 & 28.18 & \multirow{2}{*}{2.213} & \multirow{2}{*}{0.35} \\
\hline & Non-faller & 32.77 & 34.68 & & \\
\hline
\end{tabular}

Note-OSFS: Obstacle Single leg Forward Step; ES: Effect size; OSFS(F): Forward phase; OSFS(R): Returning phase.

perior in safety because it involves simple movement that even the elderly can understand easily.

Fallers were inferior in the OSFS (Obstacle single leg forward step) test to non-fallers. The 10-meter walk time and one-leg standing time with eyes open also showed similar results. Mano reported the following: a cause of walking speed decline depends on shortening of a stride length and a decrease of pace, and the former attributes this to slight bending of the knees, a small flexion angle of hip joints, and a small dorsiflexion angle of ankles when heels contact the ground. It is a so-called "shuffle" (a senile walk) (Mano, 2008). Both single and double stance phases become longer, and kicking out power also decreases with a leg strength decrease. According to physiological studies (Vandervoot and Hays, 1989; Era and Heikkinen, 1985; Gottasdanker, 1982), a cause of falls among the elderly is a decrease in consciousness-motor system function which contributes to posture and balance maintenance during walking in old age. Hence, a decline in physical fitness factors such as walk ability, balance ability etc. is also considered to be important in screening the elderly's fall risk.

The OSFS test as well as the 10-meter walk and one-leg standing time tests showed that the fallers are inferior to the non-fallers. In this connection, the former effect size was equal or somewhat larger than the two later ones. The OSFS test was constructed by considering the following four standpoints; 1 . When almost falling, the elderly step forward to one step to keep their base of support (BOS) and prevent fall. 2. A decrease in leg strength and balance ability as well as the range of motion (ROM) of the hip, knee and ankle joints are connected with factors related to fall. 3 . The elderly fall frequently by tripping over an obstacle or a step. 4 . The test for screening should use movements which the elderly can easily understand. Because the OSFS test demands that the elderly step over the obstacle while supporting the body with one leg and then return to the stepped leg's original position again, it may be difficult for them with decreased physical fitness as compared with the movements of walking and one-leg standing.

A significant difference was found in the OSFS-R between fallers and non-fallers but not in the OSFS-F. Therefore, a fall risk may be better explained by the movement (OSFS-R) in returning the stepped leg to the original place than the movement in stepping over an obstacle (OSFS-F). Because of decreased leg strength or the fear that one may fall forward, the elderly cannot stretch their legs sufficiently when their body is inclined forward (Ryushi, 2008). The movement that is required when the elderly step forward and return to an original position again exerts more force on the supporting leg in addition to maintaining balance. Hence, the elderly with decreased leg strength, balance ability, and ROM of leg joints may difficulty in achieving it successfully.

Mano (2008) reported that the elderly's shuffle contributes to maintaining balance during walking because a single-leg support time becomes shorter according to posture changes, and instability during direction change with age is due to a decline in sensory function and a delay in central response time. As confirmed in the movement used in this study and the previous study, the elderly's shuffle or senile walk may be a compensatory locomotor way to transfer to the next movement smoothly.

\section{Conclusions}

In conclusion, the OSFS test can discriminate between the fallers and non-fallers as well as 10meter walk time and one-leg standing time tests. Hence, it may be effective to evaluate the elderly's fall-related physical fitness. Additionally, the OSFS-R may be a better evaluation tool than the OSFS-F.

\section{References}

Aoyagi, Y., Togo, F., Furuna, T., Nishizawa, S., Sugiura, M., Sugita, S., Matsuki, S., Kumazaki, Y., Naka, M., Inoue, S., Takamiya, T., Ono, F., Uchida, Y., \& Inokuchi, C. (2001). Research-aid report: Development of a new method for determining exercise intensity based on the walking velocity of the elderly. Tokyo: Meiji Yasuda Life foundation of Health and Welfare. (in Japanese)

Demura, S., Sato, S., \& Sugiura, H. (2010). Lower limb laterality characteristics based on the relationship between activities and individual laterality. Gazzeta Medica Italiana, 169, 181-191.

Demura, S., Shin, S., \& Yamaji, S. (2008). Sex and age differences of relationships among stepping parameters for evaluating dynamic balance in the elderly. Journal of Physiological Anthropology, 27, 207215. doi:10.2114/jpa2.27.207

Era, P., \& Heikkinen, E. (1985). Postural sway during standing and unexpected disturbance of balance in random samples of men of different ages. The Journals of Gerontology, 40, 287-295.

Gottasdanker, R. (1982). Age and simple RT. The Journals of Gerontology, 37, 342-348.

Hosoda, T. (2001). Handbook of physical therapy (2nd ed.). Tokyo: Kyodoisyo Press. (in Japanese).

Hsiao-Wecksler, E. T. (2007). Assessing quiet and perturbed balance: A review. Japanese Journal of Biomechanics in Sports and Exercise, 11, 291-302.

Mano, Y. (2008). Falls in the elderly and measurements. Tokyo: Ishiyaku Press. (in Japanese).

Rogers, M., Hedman, L. D., Johnson, M. E., Cain, T. D., \& Hanke, T. A. (2001). Lateral stability during forward-induced stepping for dynamic balance recovery in young and older adults. Journal of Ger- 
ontology Medical Sciences, 56A, 568-594.

Ryushi, T. (2008). Falls in the elderly and measurements. Tokyo: Ishiyaku Press. (in Japanese).

Saito, S., \& Muraki, S. (2010). Study on tracks and sense of feet position while stepping over an obstacle in the elderly. Ergonomics, 46, 172-179. (in Japanese). doi:10.5100/jie. 46.172

Shin, S., \& Demura, S. (2007). Effective tempo of the step test for dynamic balance ability in the elderly. Journal of Physiological Anthropology, 26, 563-567. doi:10.2114/jpa2.26.563

Shin, S., \& Demura, S. (2009a). Relationship between the step test with stipulated tempos and gait ability in the elderly. Journal of Physiological Anthropology, 28, 49-54. doi:10.2114/jpa2.28.49

Shin, S., \& Demura, S. (2009b). The relationship of age and leg strength in the step test with stipulated tempo in the elderly. Archives of Gerontology and Geriatrics, 49, 311-316. doi:10.1016/j.archger.2008.11.009

Shin, S., \& Demura, S. (2010). Comparison and age-level differences among various step tests for evaluating balance ability in the elderly. Archives of Gerontology and Geriatrics, 50, 51-54.

doi:10.1016/j.archger.2009.05.007

Suzuki T. (2003). Epidemiology and implications of falling among the elderly. Nippon Ronen Igakkai Zasshi, 40, 85-94. (In Japanese).

Suzuki, T. (2000). Questionnaire for falls assessment of elderly people and its application. Health Assessment Manual Kosei Kagaku Kenkyusho, 142-163. (in Japanese)

Tanaka, Y., Minejima, T., Yamanaka, T., Imaizumi, H., Tanaka, M., Kawai, H., \& Hayakawa, Y. (2001). Study on reaction time and movement time in lower extremity in the elderly who have and who have not fallen. Rigakuryoho kagaku, 16, 167-171. (in Japanese) doi:10.1589/rika.16.167

Tinetti, M. E. (1986). Performance oriented assessment of mobility problems in elderly patients. Journal of the American Geriatrics Society, 34, 119-126

Vandervoot, A. A., \& Hays, K. C. (1989). Plantar flexor muscle function in young and elderly women. European Journal of Applied Physiology and Occupational Physiology, 58, 389-394. doi:10.1007/BF00643514

Whitney, S. L., Marchetti, G. F., Morris, L. O., \& Sparto, P. J. (2007). The reliability and validity of the four square step test for people with balance deficits secondary to a vestibular disorder. Archives of Physical Medicine and Rehabilitation, 88, 99-104. doi:10.1016/j.apmr.2006.10.027 